\title{
Receptive Field Encoding Model for Dynamic Natural Vision
}

\author{
Fatemeh Kamali $^{1}$, Amir Abolfazl Suratgar ${ }^{*}$, Mohamad Bagher Menhaj ${ }^{1}$, Reza Abbasi Asl ${ }^{2}$ \\ ${ }^{1}$ Department of Electrical Engineering, Amirkabir University of Technology, Tehran, Iran \\ ${ }^{2}$ Department of Neurology, University of California, San Francisco, CA, USA
}

\section{Article Info:}

\section{A BSTRACT}

Introduction: Encoding models are used to predict human brain activity in response to sensory stimuli. The purpose of these models is to explain how sensory information represent in the brain. Convolutional neural networks trained by images are capable of encoding magnetic resonance imaging data of humans viewing natural images. Considering the hemodynamic response function, these networks are capable of estimating the blood oxygen level dependence of subject viewing videos without any recurrence or feedback mechanism. For this purpose, feature map extracted from the convolutional neural network and the concept of receptive field has been used for the encoding model. The main assumption of this model is that activity in each voxel encodes a spatially localized region across multiple feature maps and for each voxel and this area are fixed for all feature maps. Contribution of each feature map in the activity of each voxel is determined by the corresponding weight. Materials and Methods: In this study, three healthy volunteers watching a set of videos. This collection contains images that represent real-life visual experience. MRI and fMRI data are acquired on a 3 tesla MRI system phase-array surface coil. Results: Data revealed that human visual cortex has hierarchical structure. Earlier visual areas have a smaller receptive field size in and response to simple feature like edge, whereas higher visual areas have a larger receptive field size and response to more complex features, such as pattern. Conclusion: This model of video stimuli has a higher interpretation capacity than the previous models.

\section{Key words:}

1. Magnetic Resonance Imaging

2. Visual Cortex

3. Brain

*Corresponding Author: Amir Abolfazl Suratgar

E-mail:a-suratgar@aut.ac.ir 


\title{
مدل رمزحذارى ميدان تأثير براى بينايى طبيعى ديناميكى
}

\author{
فاطمه كمالى'، امير ابوالفضل صورتخر ':، محمدباقر منهاج'، رضا عباسى اصل' \\ 'دانشكده مهندسى برق، دانشكاه صنعتى امير كبير، تهران، ايران

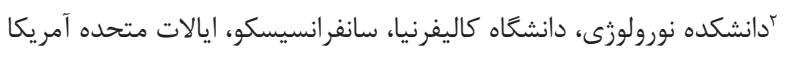

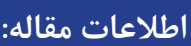

كليد وازهها:

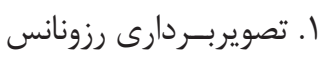

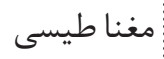

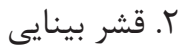

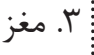

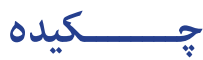

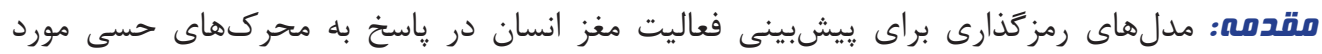

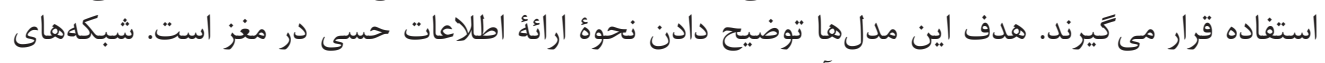

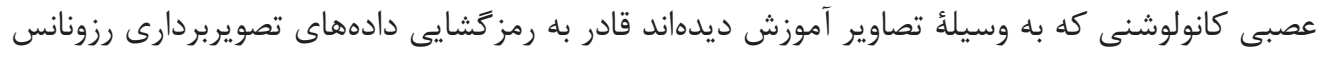

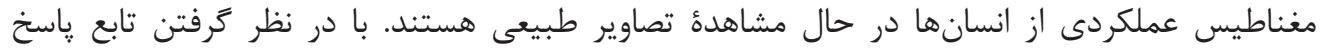

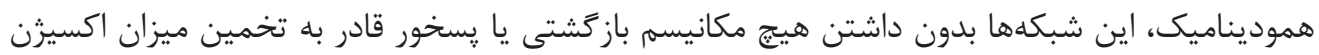

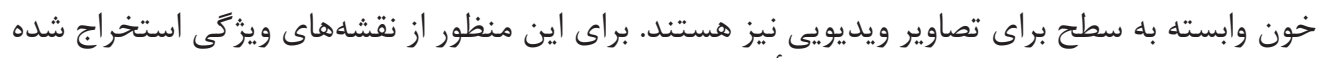

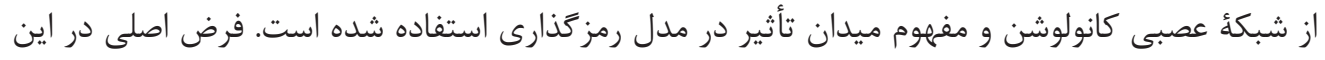

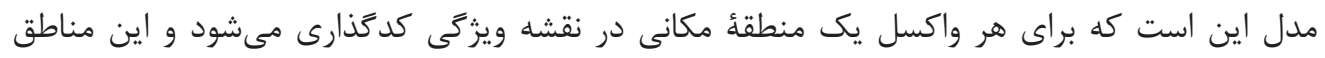

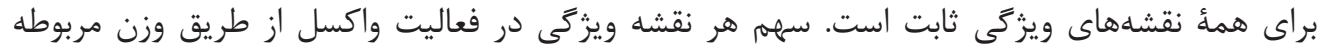

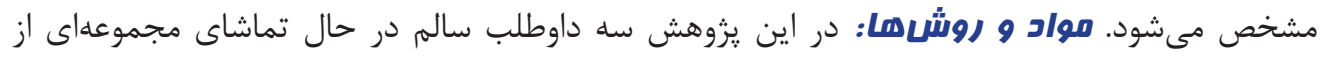

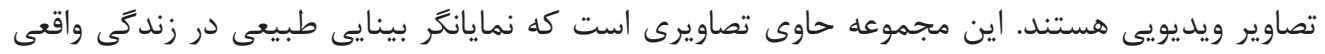

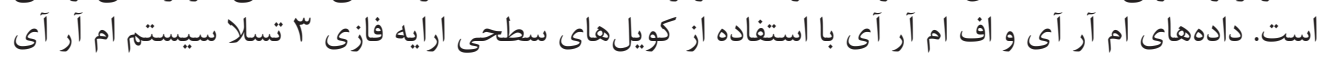

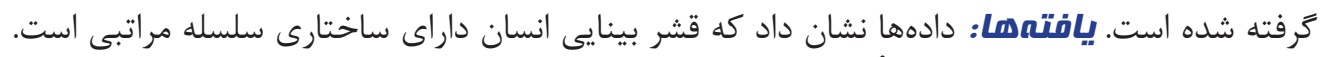

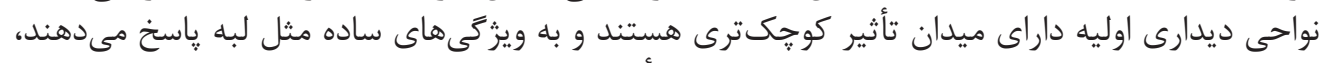

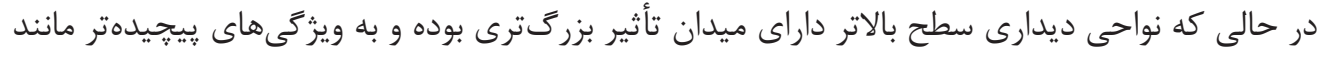

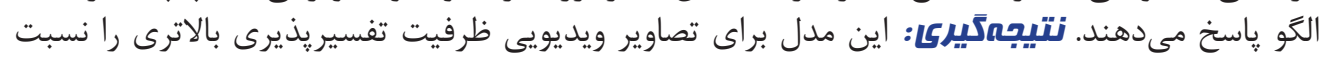
به مدل لماى بيشين دارد. 


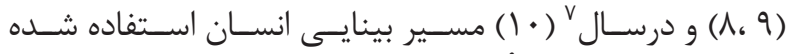
مقدمه

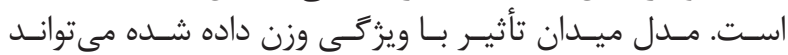

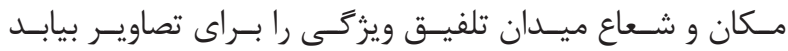

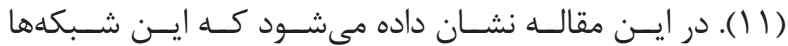

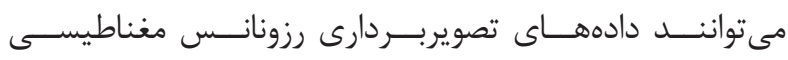

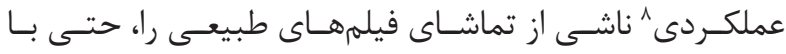

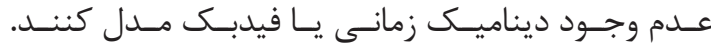

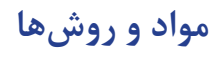

مجموعه دادهها

ســه داوطلـب سـالم در ايـن مطالعــه شـركت كردهانــد. هــر

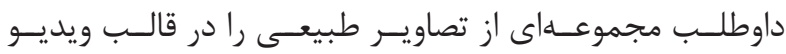

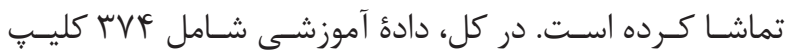

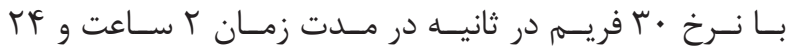

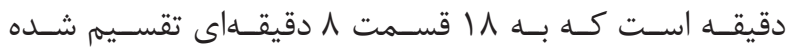

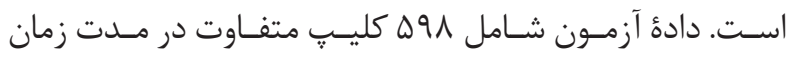

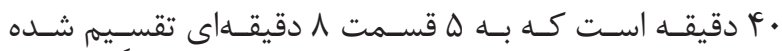

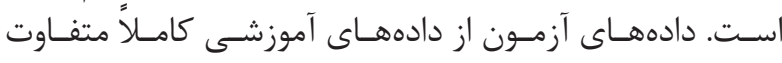

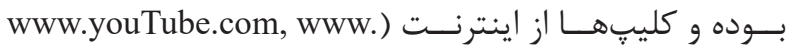
(videoblocks.com

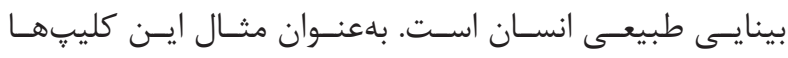

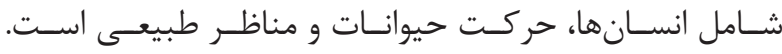

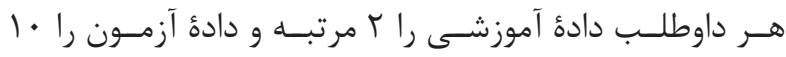

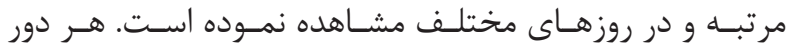

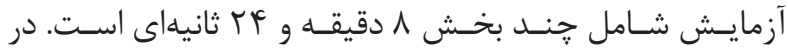

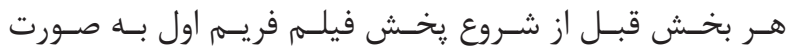

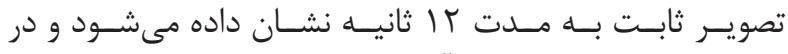

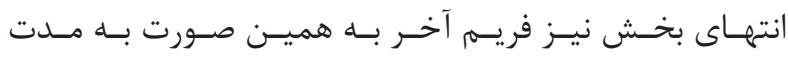

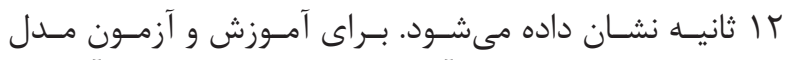

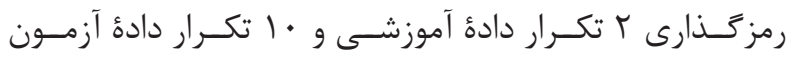

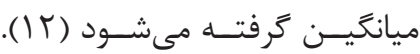

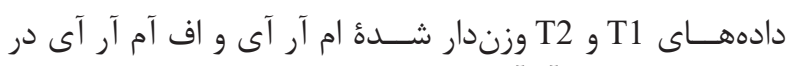

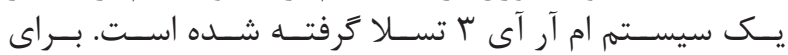

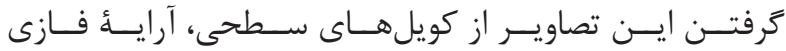

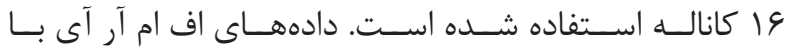

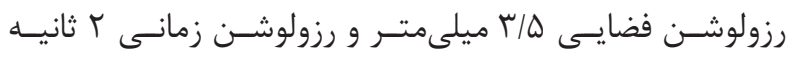

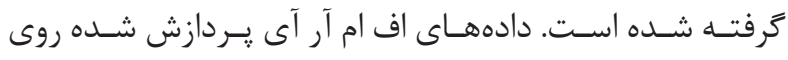

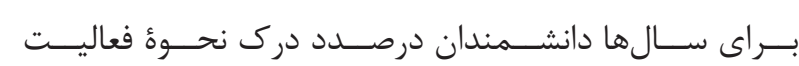

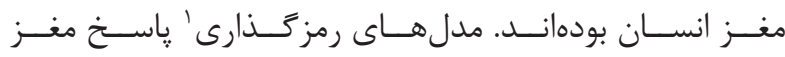

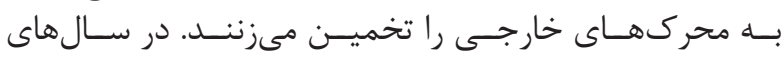

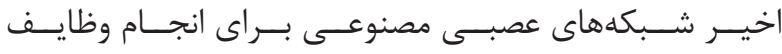

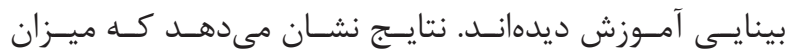

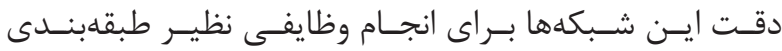

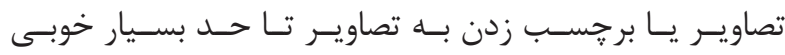

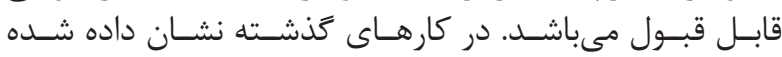

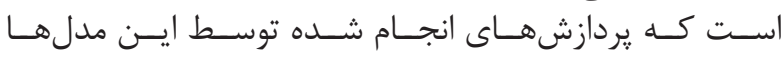

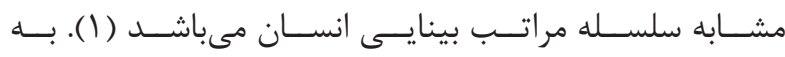

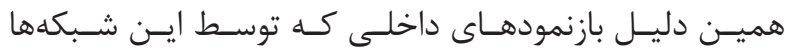

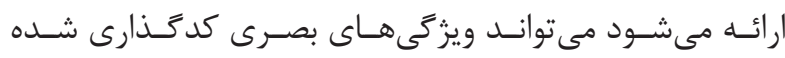

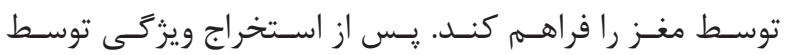

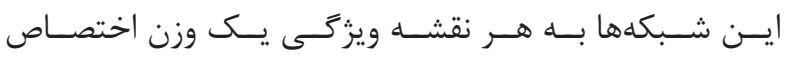

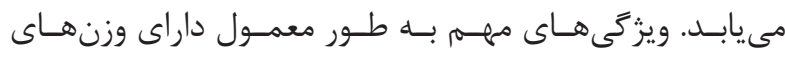

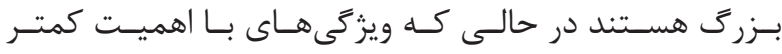

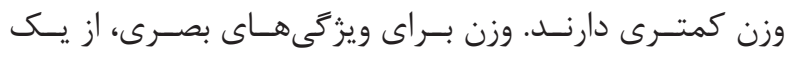

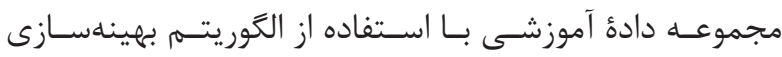

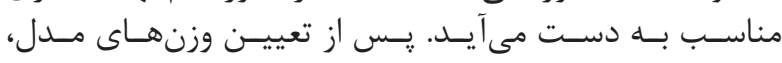

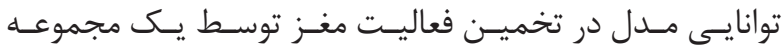

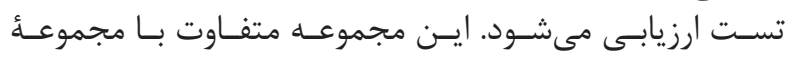

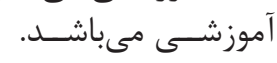

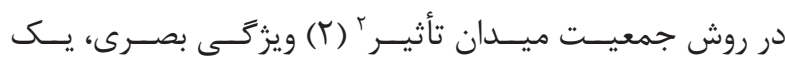

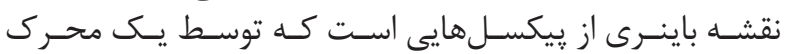

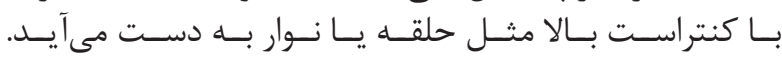

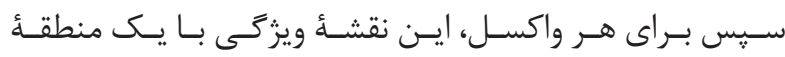

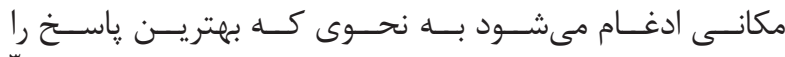

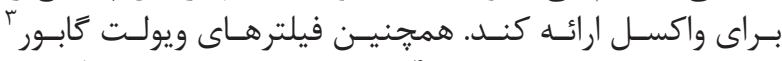

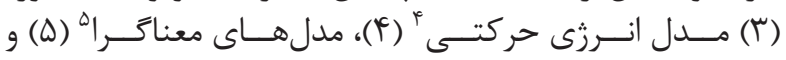

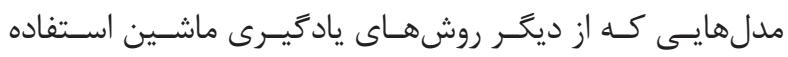

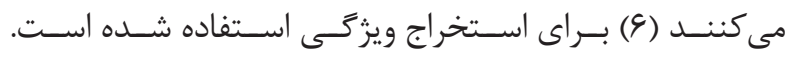

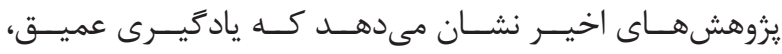

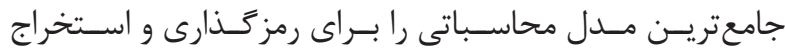

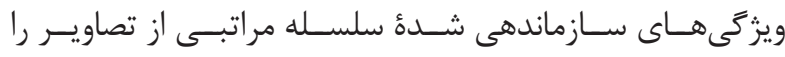

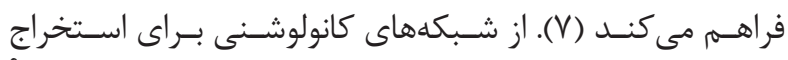

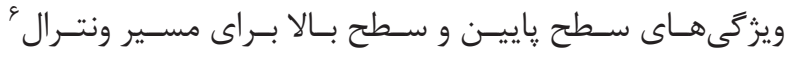

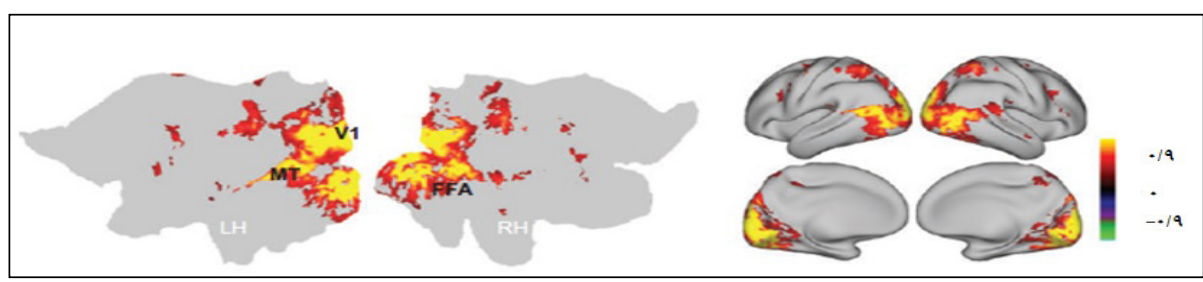

تصوير ا- همبستَى متقابل بين سيكنالهاى اف ام آر آى به دست آمده در طى دو مرحله تكرار فيلم آموزشى (r).

${ }^{1}$ Encoding model

${ }^{2}$ Receptive field

${ }^{3}$ Gabor wavelet

${ }^{4}$ Motion energy model

\author{
${ }^{5}$ Semantic model \\ ${ }^{6}$ Ventral \\ ${ }^{7}$ Dorsal \\ ${ }^{8}$ Functional magnetic resonance imaging
}




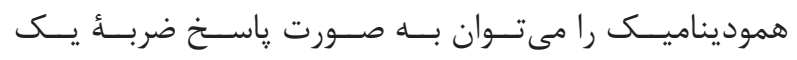

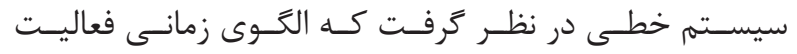

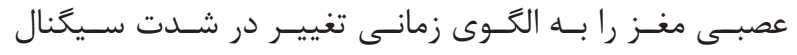

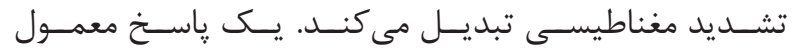

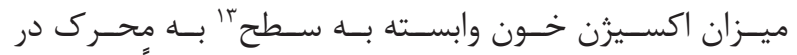

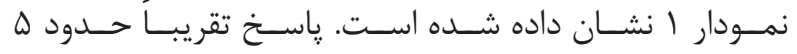

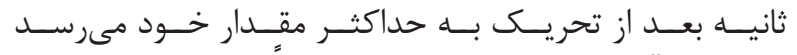

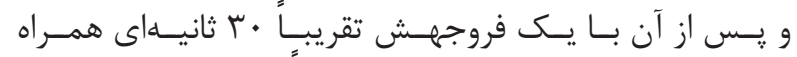

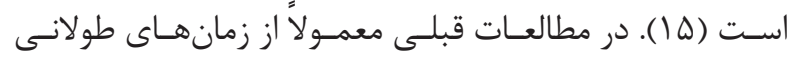

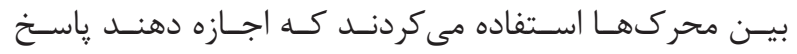

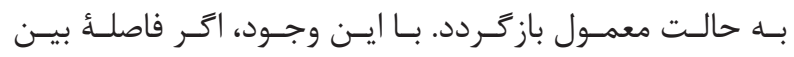

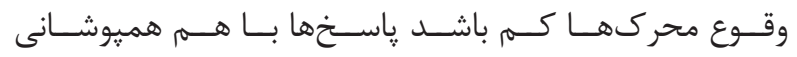

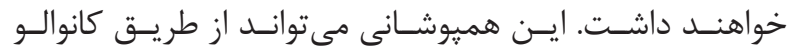

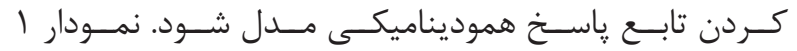

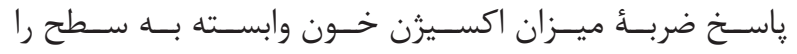

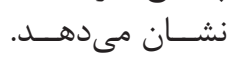

$$
\text { مدل رمزحَّارى ميدان تأثيبر }
$$

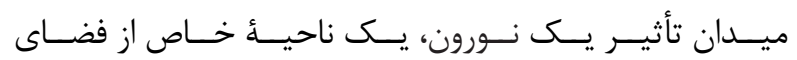

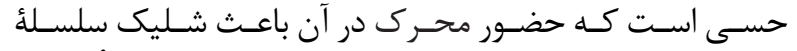

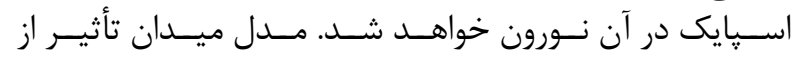

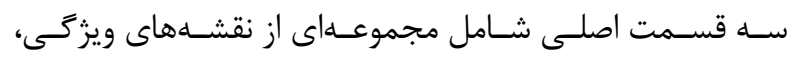

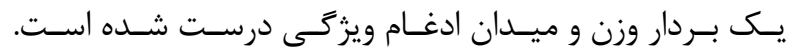

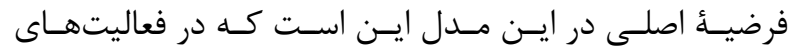

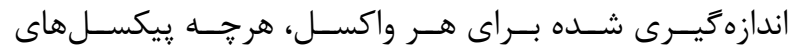

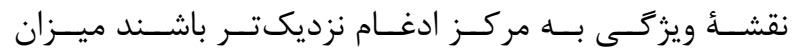

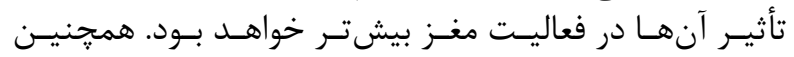

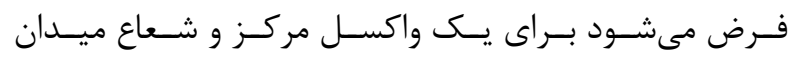

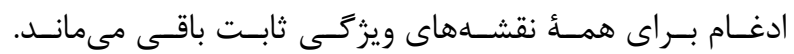

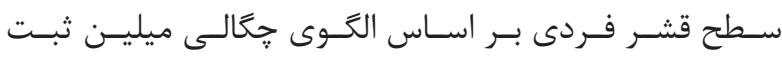

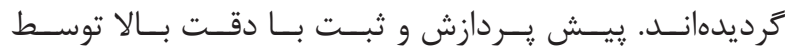

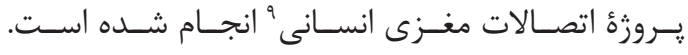

$$
\text { مرورى برشبكه هاى كانولوشنى }
$$

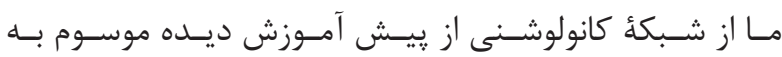

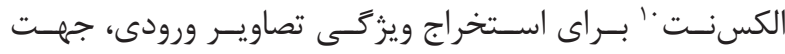

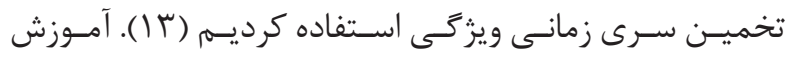

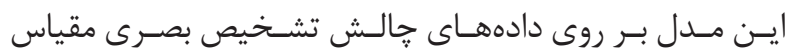

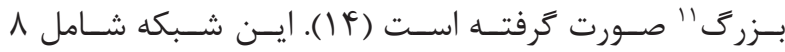

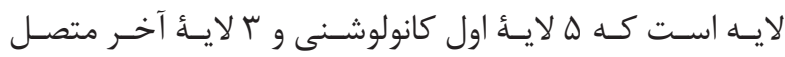

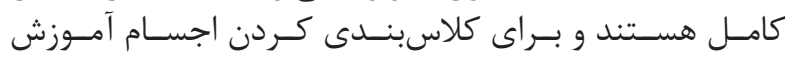

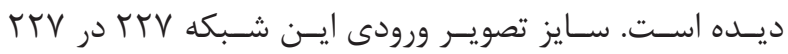

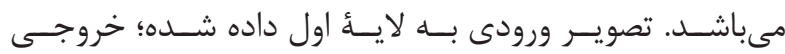

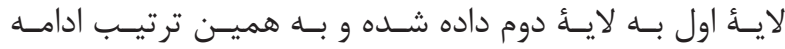

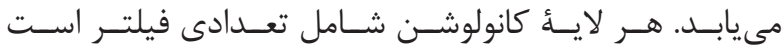

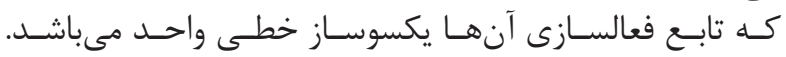

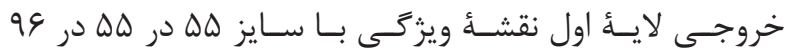

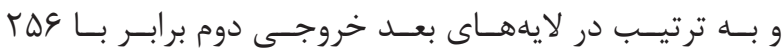

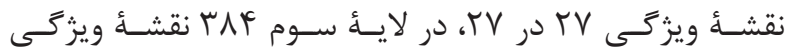

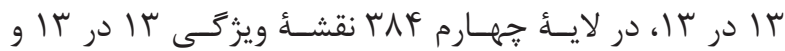

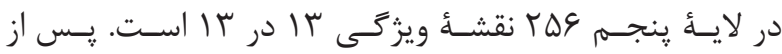

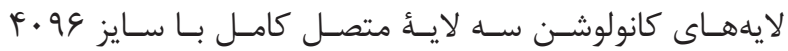
،

مرورى بر تابع پاسخ هموديناميك

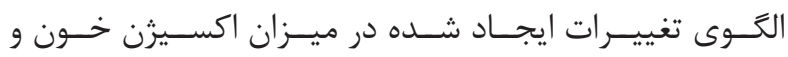

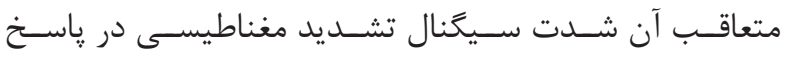

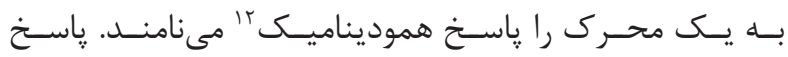
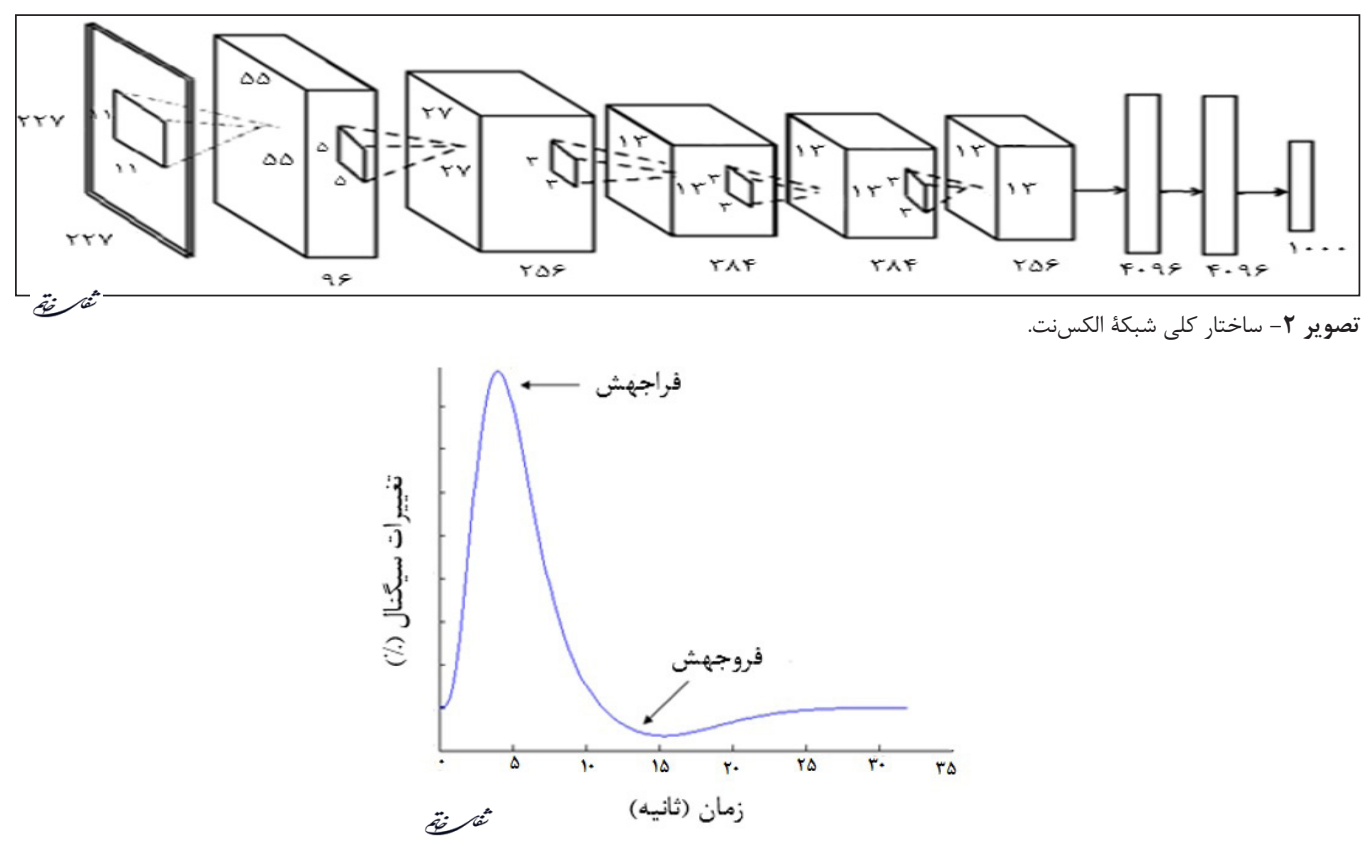

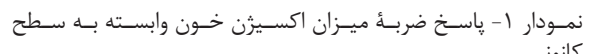

كانونسى.

${ }^{9}$ Human connectome project

${ }^{10}$ Alex net

${ }^{11}$ Large scale visual recognition challenge
${ }^{12}$ Hemodynamic response function

${ }^{13}$ Blood oxygen level dependence 


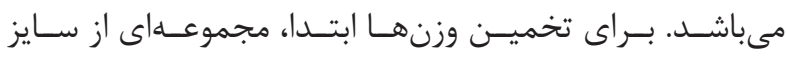

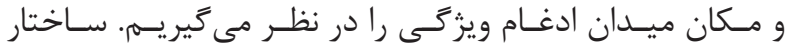

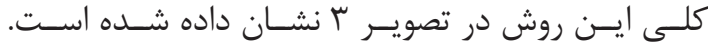

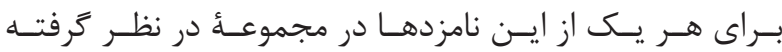

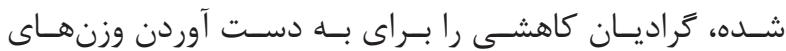

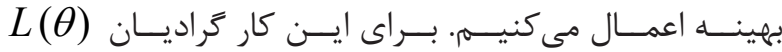

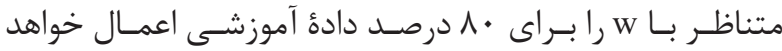

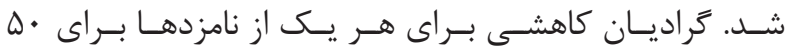

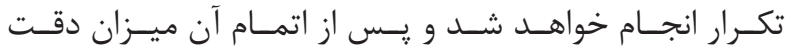

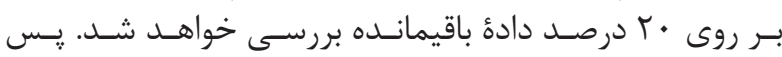

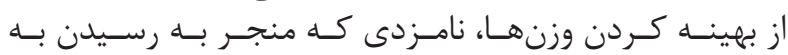

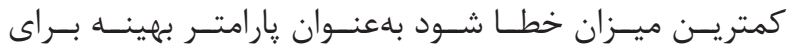

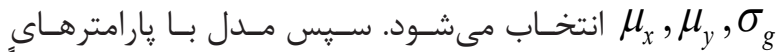

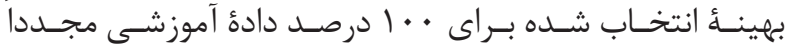

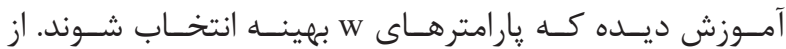

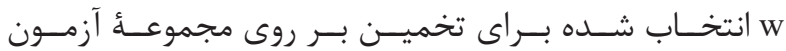
اســتفاده مى كنيـــم.

بافته ها

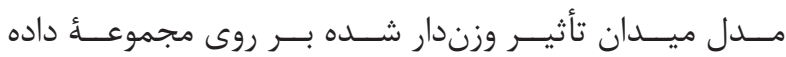

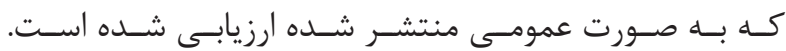

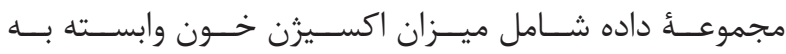

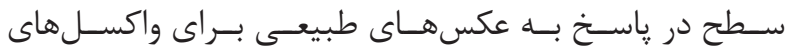
نواحسى ل

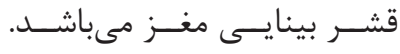

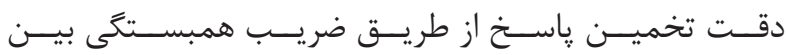

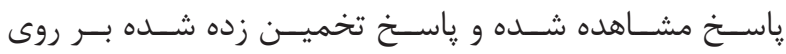

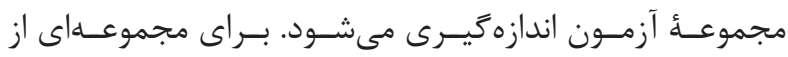

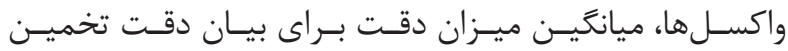

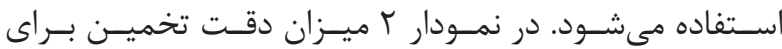

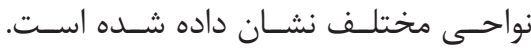

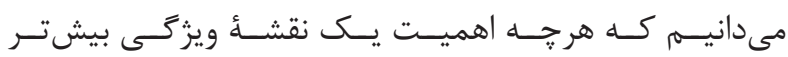

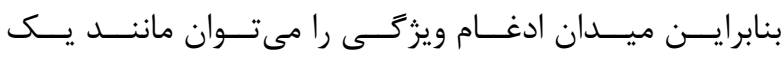

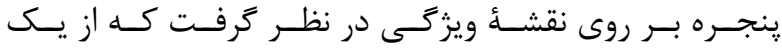

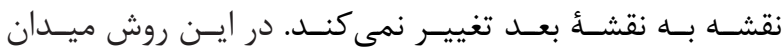

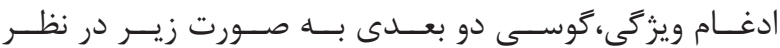

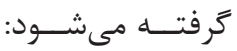
$g\left(x, y ; \mu_{x}, \mu_{y}, \sigma_{g}\right)=\frac{1}{\sqrt{2 \pi \sigma_{g}}} \exp \left[-\frac{\left(x-\mu_{x}\right)^{2}+\left(y-\mu_{y}\right)^{2}}{2 \sigma_{g}{ }^{2}}\right]$

كـهـ در فرمـول فـوق، (

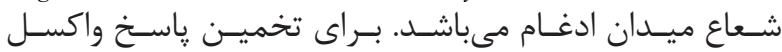

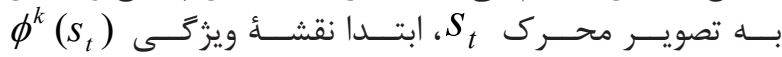

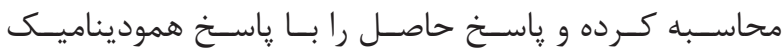

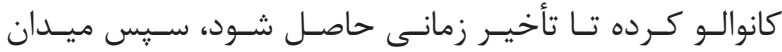

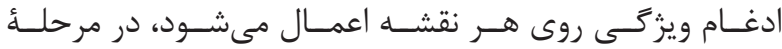

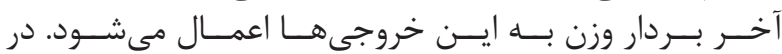
واقـع داريسهم: $\hat{r}_{t}=\sum_{k}^{K} w_{k} \int_{-D / 2}^{D / 2} \int_{-D / 2}^{D / 2} g\left(x, y ; \mu_{x}, \mu_{y}, \sigma_{g}\right)\left(h(t) * \phi_{i(x) j(y)}^{k}\left(s_{t}\right)\right) d x d y$

كـهه در ايسن فرمـول،

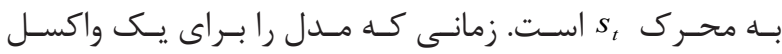

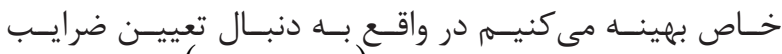

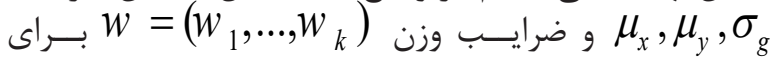

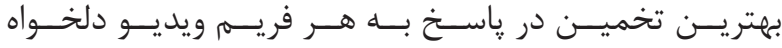

هســتيم.

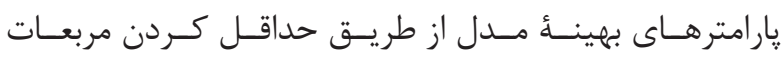

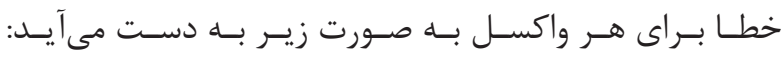

$$
E(\theta)=\sum_{t}\left(r_{t}-\hat{r}_{t}(\theta)\right)^{2}
$$

در فرمــول بــالا، S اسـت و $S_{t}$

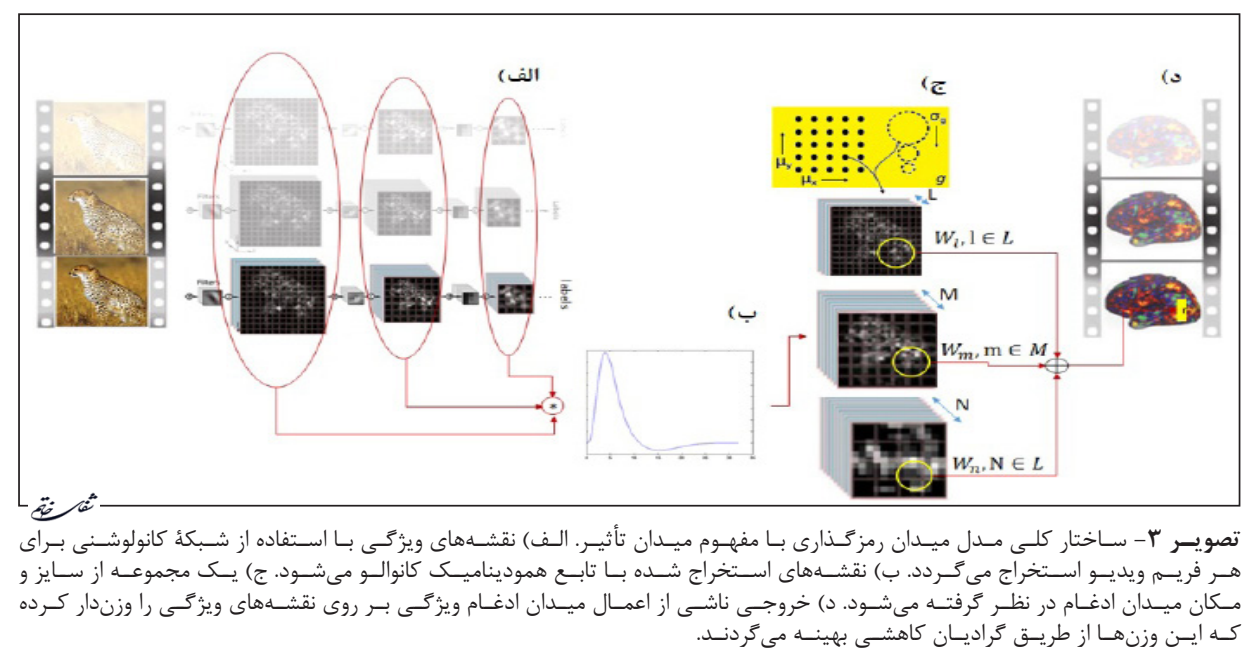

${ }^{14}$ Lateral occipital

${ }^{15}$ Middle temporal 


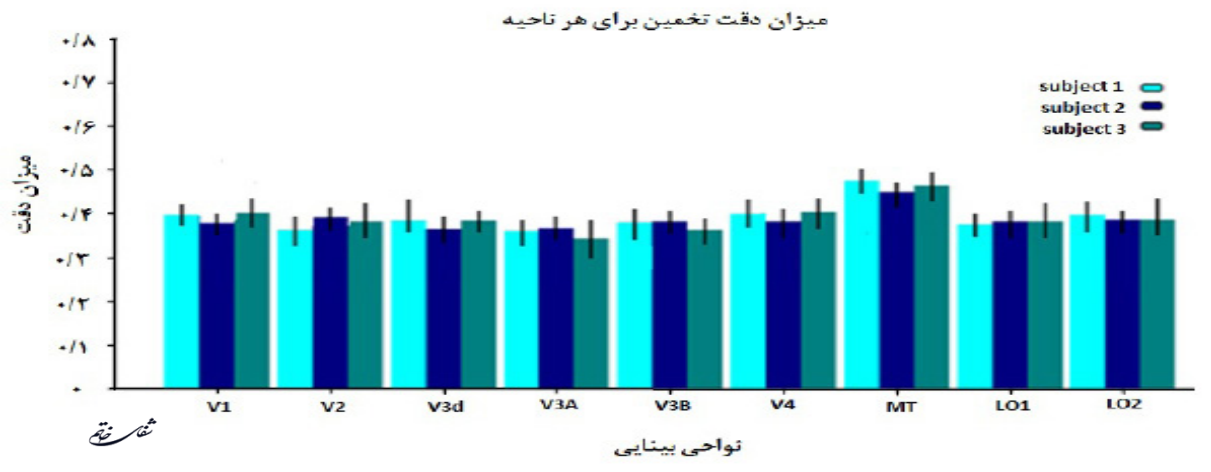

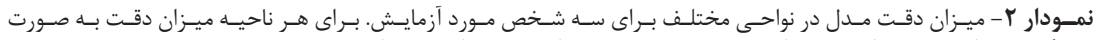

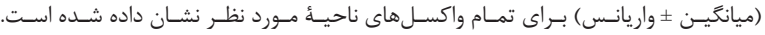

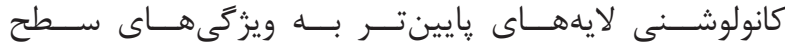

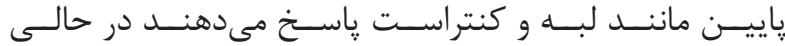

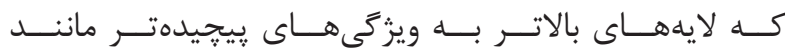

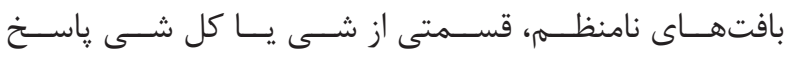

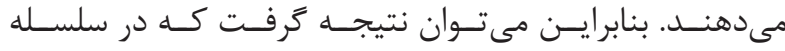

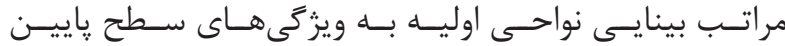

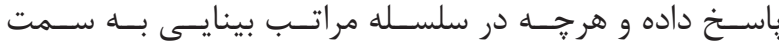

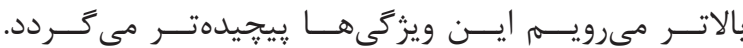

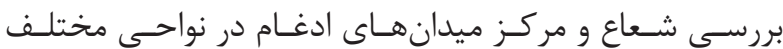

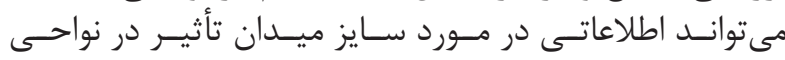

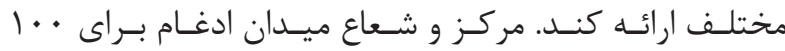

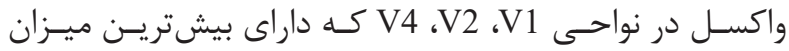

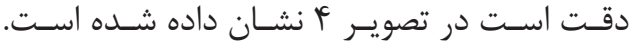

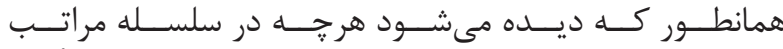

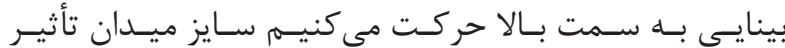

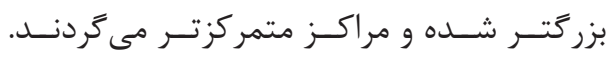

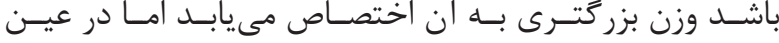

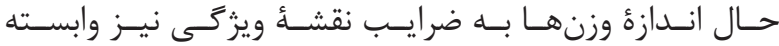

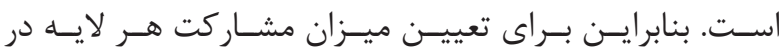

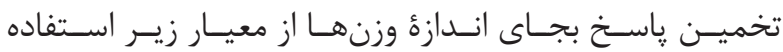
مى كنيــام.

$$
\rho_{1}=\frac{\operatorname{cov}\left(\widetilde{r}_{l} \text { 国 } r\right)_{t}}{\sqrt{\operatorname{var}(\tilde{r})_{t} \operatorname{var}(r)_{t}}}
$$

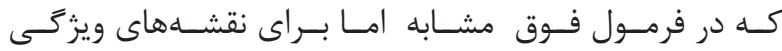

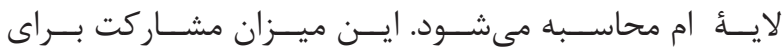

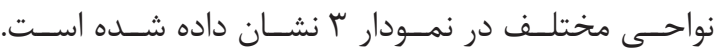

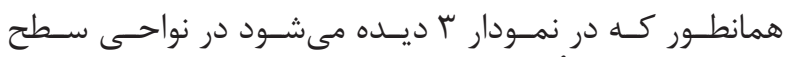

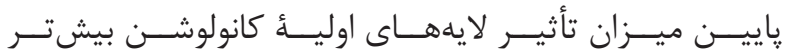

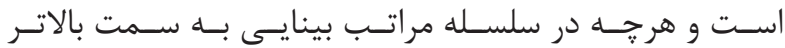

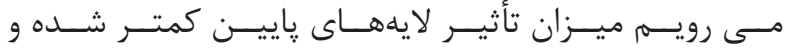

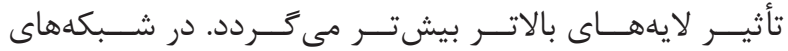
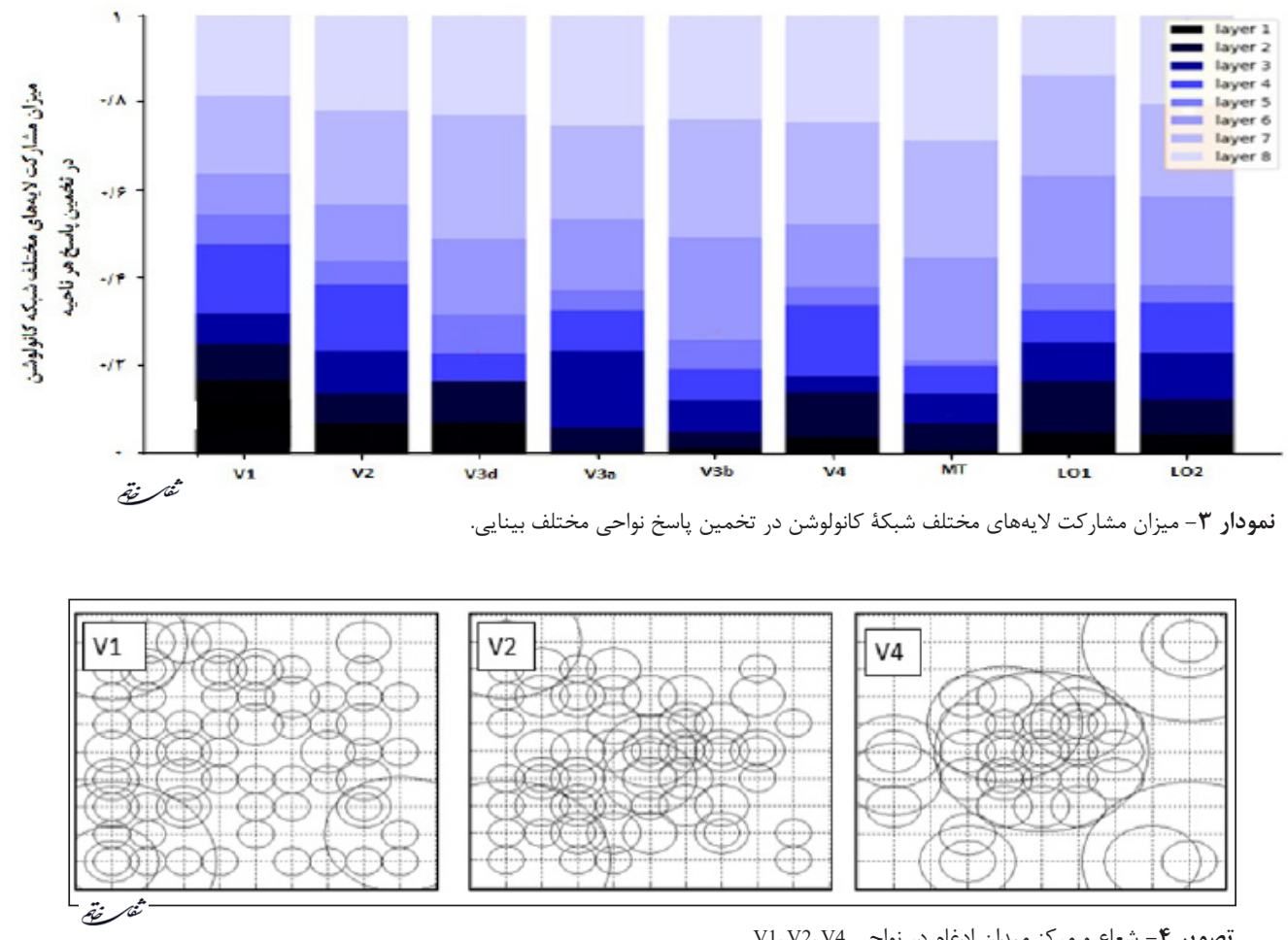

تصوير F- شعاع و مركز ميدان ادغام در نواحى V1، V2، V4. 


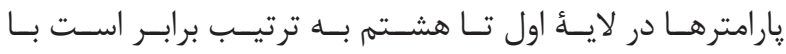

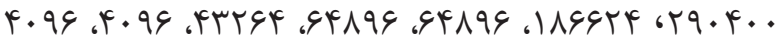

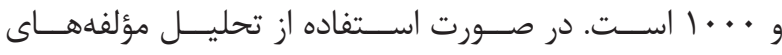

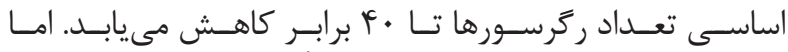

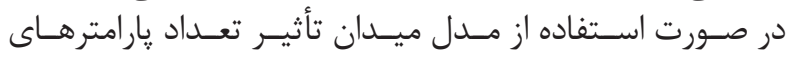

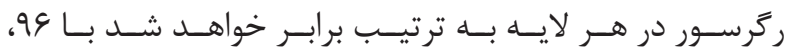
و

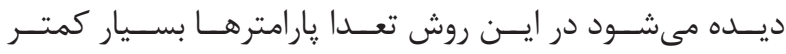

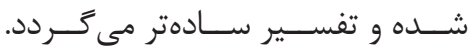

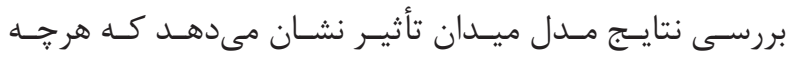

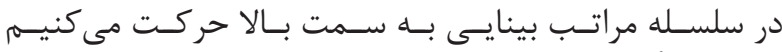

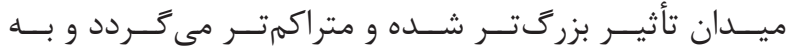

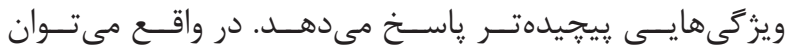

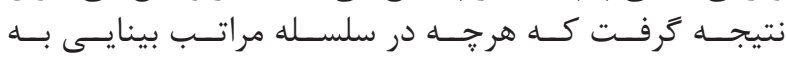

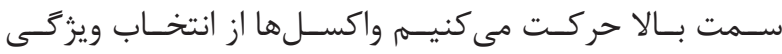

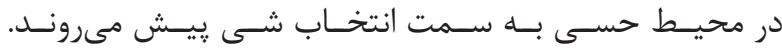

1. Güçlü U, van Gerven MA. Deep neural networks reveal a gradient in the complexity of neural representations across the ventral stream. J Neurosci. 2015; 35(27): 10005-14.

2. Dumoulin SO, Wandell BA. Population receptive field estimates in human visual cortex. Neuroimage. 2008; 39(2): 647-60.

3. Kay KN, Naselaris T, Prenger RJ, Gallant JL. Identifying natural images from human brain activity. Nature. 2008; 452(7185): 352-5.

4. Nishimoto S, Vu AT, Naselaris T, Benjamini Y, Yu $\mathrm{B}$, Gallant JL. Reconstructing visual experiences from brain activity evoked by natural movies. Curr Biol. 2011; 21(19): 1641-6.

5. Naselaris T, Stansbury DE, Gallant JL. Cortical representation of animate and inanimate objects in complex natural scenes. J Physiol Paris. 2012; 106(5-6): 239-49.

6. Stansbury DE, Naselaris T, Gallant JL. Natural scene statistics account for the representation of scene categories in human visual cortex. Neuron. 2013; 79(5): 1025-34.

7. LeCun Y, Bengio Y, Hinton G. Deep learning. Nature. 2015; 521(7553): 436.

8. Cichy RM, Khosla A, Pantazis D, Torralba A, Oliva A. Deep neural networks predict hierarchical spatio-

$$
\text { بحث و نتيجه كيرى }
$$

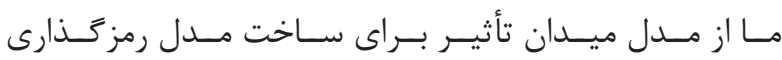

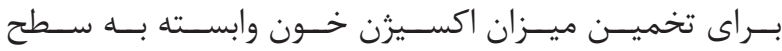

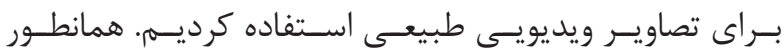

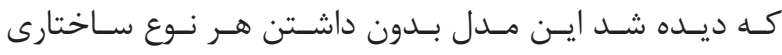

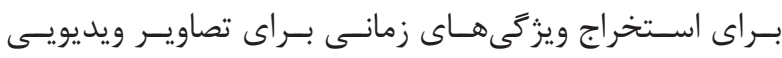

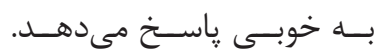

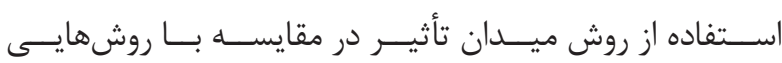

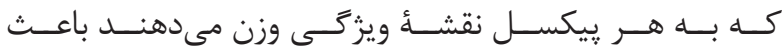

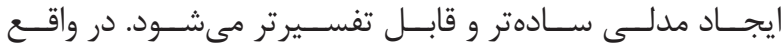

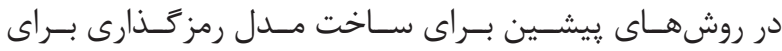

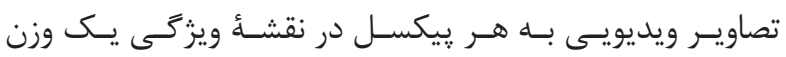

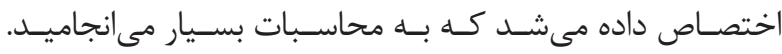

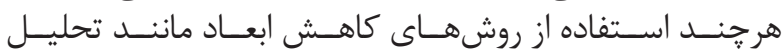

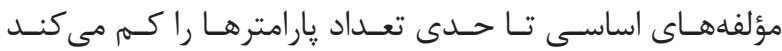

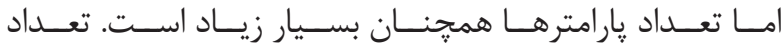

منابع

temporal cortical dynamics of human visual object recognition. arXiv preprint arXiv:1601.02970. 2016.

9. Agrawal P, Stansbury D, Malik J, Gallant JL. Pixels to voxels: modeling visual representation in the human brain. arXiv preprint arXiv:1407.5104. 2014.

10. Khaligh-Razavi SM, Kriegeskorte N. Deep supervised, but not unsupervised, models may explain IT cortical representation. PLoS Comput Biol. 2014; 10(11): e1003915. doi: 10.1371/journal.pcbi.1003915.

11. St-Yves G, Naselaris T. The feature-weighted receptive field: an interpretable encoding model for complex feature spaces. NeuroImage. 2018; 180: 188-202.

12. Wen H, Shi J, Zhang Y, Lu KH, Cao J, Liu Z. Neural encoding and decoding with deep learning for dynamic natural vision. Cerebral Cortex. 2017; 28(12): 4136-60.

13. Krizhevsky A, Sutskever I, Hinton GE. Imagenet classification with deep convolutional neural networks. Advances in Neural Information Processing Systems. 2012; 25(2): 1-9.

14. Russakovsky O, Deng J, Su H, Krause J, Satheesh $\mathrm{S}$, Ma S, et al. Imagenet large scale visual recognition challenge. International Journal of Computer Vision. 2015; 115(3): 211-52.

15. Henson R, Friston K. Convolution models for fMRI. statistical parametric mapping: The analysis of functional brain images. 2007: 178-92. 